\title{
A New Atlas of the City of Irkutsk
}

\author{
L. A. Plastinin \\ National Research Irkitsk State Technical University, Irkutsk, Russia \\ e-mail:info@istu.edu \\ Received January 20, 2012
}

DOI: $10.1134 / \mathrm{S} 1875372812010179$

In commemoration of the $350^{\text {th }}$ Anniversary of the city of Irkutsk, a new fundamental atlas (Irkutsk Development Atlas, Irkutsk: Izd-vo In-ta geografii SO RAN, 2011) was published by V.B. Sochava Institute of Geography, Siberian Branch, Russian Academy of Sciences. This atlas was made in cooperation with the research institutes and higher educational establishments of Irkutsk and with the support of the City Administration.

This new atlas contains 62 thematic maps of the city and its neighboring territories, which harmoniously supplemented with the text materials, many diagrams and photographs. The atlas has been subdivided into five sections: introductory section, natural conditions, economic and infrastructural potential, social and demographic potential, current status of the urban environment.

The introductory section outlines the geographical location, history and development of Irkutsk. In addition to the traditional content of historical maps, this section contains two unique cartographic plots showing the growth of the city territory and of its suburbs in the process of development of Irkutsk.

Section 1 includes maps displaying the natural conditions of the city and its immediate vicinities. The maps are arranged in accordance to a conventional scheme: the physical map, geology, geomorphology, climate, surface waters, soils, fauna, vegetation, and landscapes. It is for the first time that such a sequence of maps for the natural conditions is displayed at a large scale.

Section 2 is devoted to the city's economic and infrastructural potential. The section begins with an original land use map, followed by maps displaying the development of the overall economic potential and its components (industrial production, civil construction, and the housing sector), changes in elements of the infrastructural potential (quantity and quality of transport service, forecasting of traffic streams, transport accident rate, exterior transportation, and tertiary industries), and the formation processes of Irkutsk urban agglomeration.
Section 3 characterizes the demographic and social potential of the city. This section includes the maps showing the population number, the density dynamics, the situation in the municipal, government and private health care systems, with the medication supply, the system of higher and secondary education, the situation with the scientific institutions. The political activity of the population and the confessional situation are displayed on the maps for the first time. Considerable attention is devoted to the processes of a further development of culture, sports and tourism, and to archaeological and historical-cultural heritage sites.

The present state of the urban environment is outlined in section 4 . This section begins with schematic maps showing the fundamental types and density of urban development. These maps demonstrate: the atmospheric air and water pollution; the degree of impact caused to the vegetation; the situation with soil pollution; the ecologic-biochemical state of the soils; ecological risks to the population. This section also contains the maps showing the development of a network of specially protected territories.

And finally, this atlas presents the general layout of Irkutsk urban-type development.

The atlas of Irkutsk forms a part of the main fundamental cartographic project, i.e. the atlas entitled "The Baikal region: Its nature and the society". It serves as the indispensable element of a special-purpose atlas-based information system (AIS) - a software package intended for implementation of scientific and technological methods and facilities for computeraided acquisition, storage, real-time conversion and display of heterogeneous territorial information in the cartographic form and also in some other forms convenient for end users. The main advantages of this AIS include: the systems approach, the goal-oriented nature and the multivariable character of a generation of fundamental digital maps. Such a combination of the advantages has been achieved at the expense of application of contemporary information technologies with respect to databases containing geographical, cartographical and other data. 